\title{
Emergency management of puerperal breast abscess
}

\author{
Jeffrey Forris Beecham Chick • Nikunj Rashmikant Chauhan • \\ Lauren Armen Polen
}

Received: 27 March 2012/Accepted: 21 June 2012/Published online: 8 July 2012

(c) SIMI 2012

\section{Case report}

A 38-year-old woman, who was 1 month postpartum, presented with left breast pain. The patient reported a 5-day history of stabbing left breast pain, erythema, and tenderness. She had been breast feeding and pumping breast milk without difficulty, but recently noted blood, mucous, and purulent material within the pumped milk. The patient denied fevers, chills, breast trauma, a palpable mass, or right breast abnormalities. Ultrasound of the left breast demonstrated a $4.7 \times 2.1 \mathrm{~cm}$ complex fluid collection with associated skin thickening, consistent with left breast cellulitis, mastitis, and a breast abscess (Fig. 1). Surgical consultation was obtained, and a trial of ultrasound-guided aspiration of the left breast abscess was completed (Fig. 2) that yielded $12 \mathrm{ml}$ of purulent fluid. Aspirated samples were evaluated by microbiology with cultures positive for methicillin-resistant Staphylococcus aureus. Trimethoprim and sulfamethoxazole were initiated for 7 days. Repeat ultrasound of the left breast demonstrated complete resolution of the left breast abscess (Fig. 3). On return visit, the patient reported decreased pain, swelling, and drainage from the left breast, and has subsequently been able to resume left breast feeding and pumping.

\section{J. F. B. Chick $(\bowtie) \cdot$ N. R. Chauhan}

Department of Radiology, Brigham and Women's Hospital, Harvard Medical School, 75 Francis Street, Boston, MA 02115, USA

e-mail: jchick@partners.org

L. A. Polen

Department of Emergency Medicine, Stanford Hospital and Clinics, Stanford University School of Medicine, Stanford, CA 94305, USA

\section{Discussion}

Cellulitis, mastitis, and breast abscesses exist along a continuum, with breast abscesses occurring in 5-11\% of lactating women with infectious mastitis [1]. Most breast abscesses develop secondarily to skin contamination with S. aureus, Staphylococcus epidermidis, Streptococcus pyogenes, Peptostreptococcus, or Bacteroides [1]. Common symptoms of breast abscesses, as illustrated by this case, include breast pain, erythema, tenderness, and increased breast temperature with or without fever or a palpable breast mass. Ultrasonography is the imaging modality of choice due to its availability, painless use, ability to conduct repeat examinations during the course of therapy, and its ability to guide percutaneous drainage [2]. In cases of confirmed breast abscesses, ultrasonography usually demonstrates a multiloculated hypoechoic fluid collection of variable shape and size with a thickened periphery and increased vascularity. According to recently published 2011 algorithm, a trial of ultrasound guided drainage is recommended for all fluid collections suggesting an abscess, regardless of size, which occur during the breastfeeding period, are caused by S. aureus, and lack a multiloculated structure. The greatest success is achieved with abscesses that are $<3 \mathrm{~cm}$ in diameter [3, 4]. Abscesses that are multiloculated or larger than $3 \mathrm{~cm}$ in diameter may fail ultrasound-guided drainage; thus, in such instances, early surgical evaluation for incision and drainage is strongly recommended [4]. In addition to ultrasound-guided drainage, all suspected breast abscesses should be managed with antibiotics including cloxacillin $500 \mathrm{mg}$ four times daily, clindamycin $300 \mathrm{mg}$ four times daily, erythromycin $500 \mathrm{mg}$ three times daily, or cefazolin $500 \mathrm{mg}$ four times daily for a total of 7-10 days [4, 5]. Breast feeding should be continued throughout the treatment period and only 


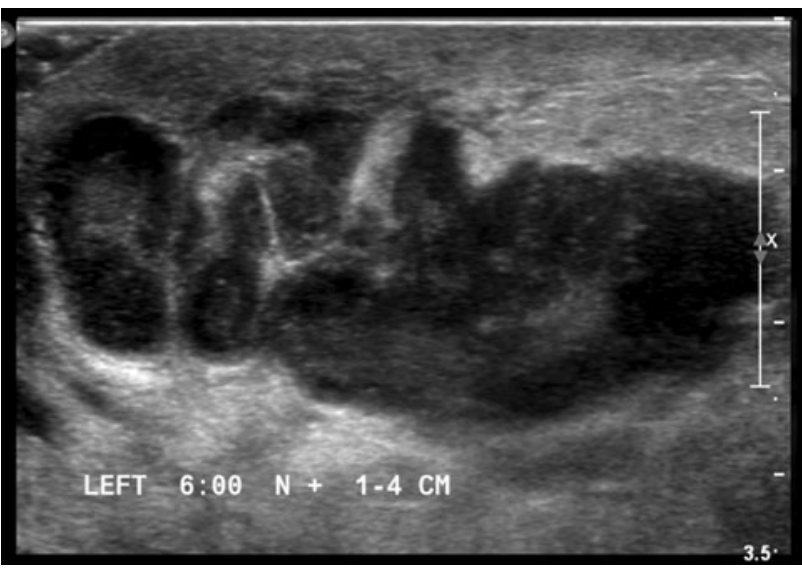

Fig. 1 Single gray-scale ultrasound image of the left breast at 6 o'clock, $4 \mathrm{~cm}$ from the nipple demonstrating a $4.7 \times 2.1 \mathrm{~cm}$ complex fluid collection with associated skin thickening, consistent with cellulitis, mastitis, and a large breast abscess

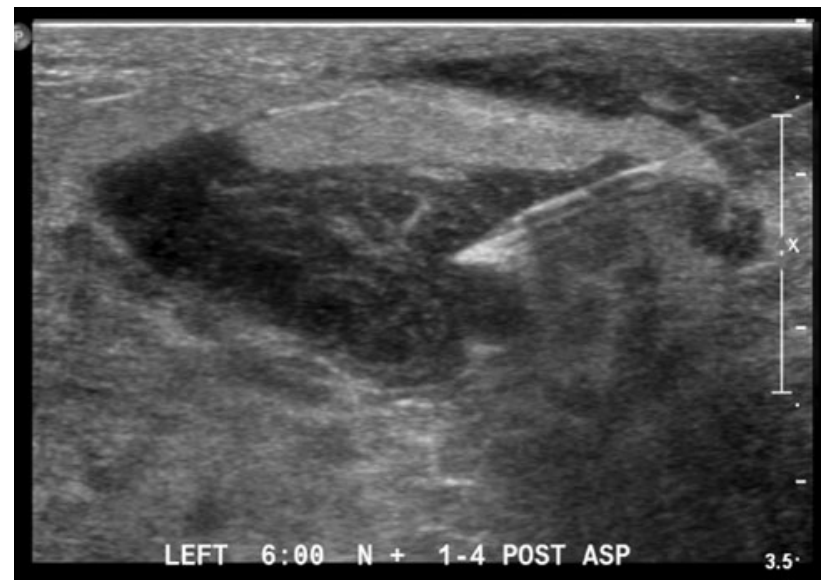

Fig. 2 Single gray-scale ultrasound image of the left breast abscess aspiration demonstrating aspiration of the left breast fluid collection using an 18 gauge needle with a lateral approach. Aspiration yielded $12 \mathrm{ml}$ of purulent fluid

discontinued when using antibiotics that are contraindicated for newborns (ciprofloxacin, tetracycline, or chloramphenicol), or if surgical incision and drainage is completed $[4,5]$. In the rare cases in which breast abscess is clinically suspected, but initial ultrasound fails to demonstrate a defined fluid collection, evaluation for

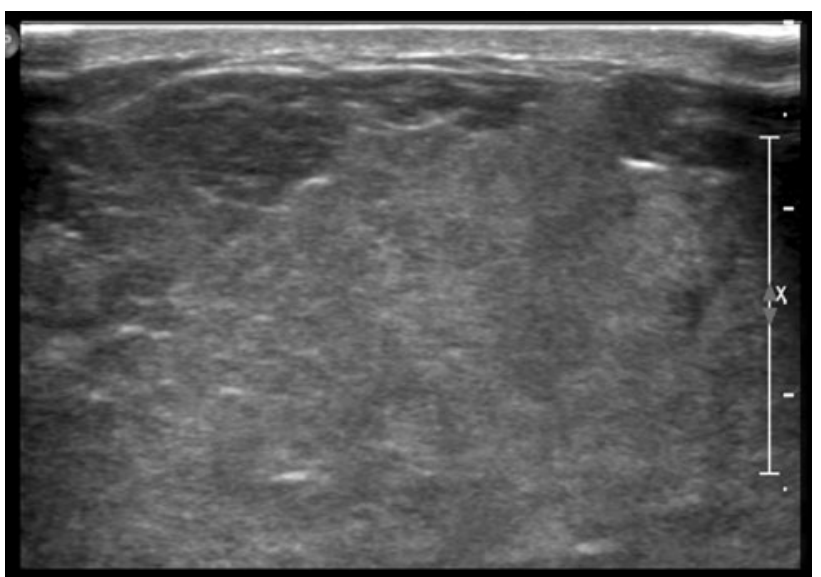

Fig. 3 Single gray-scale ultrasound image of the left breast at 6 o'clock, $4 \mathrm{~cm}$ from the nipple in the region of the previous breast abscess demonstrating resolution of previous breast abscess with residual mild breast edema

inflammatory carcinoma should be completed with mammography or breast biopsy [6].

\section{Conflict of interest None.}

\section{References}

1. Dixon JM (2006) Breast infection. In: Dixon JM (ed) ABC of breast diseases, 3rd edn. Blackwell, Oxford, pp 19-23

2. Christensen AF, Al-Suliman N, Nielsen KR et al (2005) Ultrasound-guided drainage of breast abscesses: results in 151 patients. Br J Radiol 78(927):186-188

3. Imperiale A, Zandrino F, Calabrese M, Parodi G, Massa T (2001) Abscesses of the breast: US-guided serial percutaneous aspiration and local antibiotic therapy after unsuccessful systemic antibiotic therapy. Acta Radiol 42(2):161-165

4. Trop I, Dugas A, David J, El Khoury M, Boileau JF, Larouche N, Lalonde L (2011) Breast abscesses: evidence-based algorithms for diagnosis, management, and follow-up. Radiographics 31(6):16831700

5. Leborgne F (2003) Treatment of breast abscesses with sonographically guided aspiration, irrigation, and instillation of antibiotics. AJR Am J Roentgenol 181(4):1089-1091

6. Berna-Serna JD, Madrigal M, Berna-Serna JD (2004) Percutaneous management of breast abscesses: an experience of 39 cases. Ultrasound Med Biol 30(1):1-6 